\title{
Technè
}

La science au service de l'histoire de l'art et de la préservation des biens culturels

40 | 2014

Thérapéia. Polychromie et restauration de la sculpture dans l'Antiquité

\section{La Corinthe de J.-L. Gérôme, reconstitution ou libre imitation de la polychromie grecque antique?}

Jean-Léon Gérôme's Corinth, reconstruction or free imitation of Ancient Greek polychromy?

Axelle Davadie, Édouard Papet, Nathalie Pingaud, Anne-Solenn Le Hô, Agnès Cascio, Juliette Levy-Hinstin et Marie-Emmanuelle Meyohas

\section{OpenEdition}

Journals

Édition électronique

URL : https://journals.openedition.org/techne/3935

DOI : 10.4000/techne.3935

ISSN : 2534-5168

Éditeur

C2RMF

Édition imprimée

Date de publication : 26 novembre 2014

Pagination : 123-131

ISBN : 978-2-7118-6218-4

ISSN : $1254-7867$

\section{Référence électronique}

Axelle Davadie, Édouard Papet, Nathalie Pingaud, Anne-Solenn Le Hô, Agnès Cascio, Juliette LevyHinstin et Marie-Emmanuelle Meyohas, "La Corinthe de J.-L. Gérôme, reconstitution ou libre imitation de la polychromie grecque antique? », Technè [En ligne], 40 | 2014, mis en ligne le 24 juillet 2020, consulté le 08 février 2022. URL : http://journals.openedition.org/techne/3935; DOI : https://doi.org/ 10.4000/techne.3935

\section{cc)}

La revue Technè. La science au service de l'histoire de l'art et de la préservation des biens culturels est mise à disposition selon les termes de la Licence Creative Commons Attribution - Pas d'Utilisation Commerciale - Pas de Modification 4.0 International. 
Axelle Davadie

Édouard Papet

Nathalie Pingaud

Anne-Solenn Le Hô

Agnès Cascio

Juliette Levy-Hinstin

Marie-Emmanuelle Meyohas

\section{La Corinthe de J.-L. Gérôme, reconstitution ou libre imitation de la polychromie grecque antique?}

Jean-Léon Gérôme’s Corinth, reconstruction or free imitation of Ancient Greek polychromy?

Résumé. Acquise en 2008, Corinthe (1903), l’un des rares plâtres originaux de J.-L. Gérôme (1824-1904), est probablement la dernière cuvre de l'artiste, plus connu comme peintre. Afin d'en mieux comprendre les techniques de réalisation et l'état lors de l'achat, cette sculpture a fait l'objet d'une étude préalable menée en collaboration par le musée d'Orsay, les restauratrices et les services concernés du C2RMF. Cette première étude a révélé que la subtile polychromie de J.-L. Gérôme était masquée par un épais repeint du début du XX $X^{e}$ siècle. Après plusieurs tests, le choix audacieux d'un dégagement de la polychromie originale s'est imposé. Mise au jour par les restauratrices, cette polychromie permet aujourd'hui de mieux apprécier le caractère novateur de cette cuvre et fait regretter la non-localisation du marbre posthume, lui aussi polychrome.

Mots-clés. Polychromie antique, courant néo-grec, Corinthe, archéologie, J.-J. Winckelmann, D.-R. Rochette, L.-E. Decorchemont, armatures, plâtre original, amidon, aluminium, cire, bronzine, dégagement polychromie originale.
Abstract. Acquired in 2008, Corinth (1903), one of the rare original plasters made by J.-L. Gérôme (1824-1904), is probably the last work of the artist who was better known for his paintings. To obtain a clearer understanding of the production techniques involved and the condition of the statue at the time of its purchase, this sculpture underwent a preliminary study, jointly conducted by the Musée d'Orsay and the conservators and relevant departments. This initial study showed how J.-L. Gérôme's subtle polychromy was concealed by a layer of thick paint applied in the early 20 th century. After several tests, the bold decision to uncover the original polychromy was made. Refreshed by restorers, this polychromy today enables us to better appreciate the innovative character of this work and regret that the location of the posthumous marble, also in colour, remains unknown.

Keywords. Ancient polychromy, Neo-Greek trend, Corinth, archaeology, J.-J. Winckelmann, D.-R. Rochette, L.-E. Decorchemont, armatures, original plaster, starch, aluminium, wax, bronzine. Uncovering the original polychromy.
Le musée d'Orsay a choisi de faire suivre la restauration de la Corinthe, plâtre polychrome de Jean-Léon Gérôme, par le Centre de Recherche et de Restauration des musées de France, inscrivant cette opération dans une longue tradition de collaboration, depuis la publication, Sculptures en cire de l'Égypte ancienne à l'art abstrait ${ }^{1}$, jusqu'aux études sur les sculptures caricaturales en différents matériaux dues à Honoré Daumier ${ }^{2}$.

Avec ce plâtre, un nouveau domaine d'étude est ouvert aux investigations, celui de la polychromie, que Quatremère de Quincy qualifiait d'artificielle, sur un support monochrome: en cela, elle différait de celle pratiquée par Cordier, qui pouvait associer dans une même œuvre des marbres de couleur variée ${ }^{3}$.

\section{Corinthe, une œuvre du $\mathrm{XIX}^{\mathrm{e}}$ siècle?}

\section{Théorie artistique et contexte historique}

La réalisation de la Corinthe au XIX ${ }^{\mathrm{e}}$ siècle doit être replacée dans le contexte qui la vit éclore: celui du débat, apparu dès la fin du Moyen Âge, sur la couleur des sculptures au moment même où Vasari parlait de rinascità à propos des œuvres de Nicolo da Pisano, inspirées du décor d'un sarcophage romain. Avec la découverte et la fouille (à partir de 1738) des vestiges d'Herculanum puis de Pompéi en Italie, le monde des antiquaires du XVIII ${ }^{\mathrm{e}}$ siècle doit penser l'art et l'esthétique selon de nouveaux critères, nés de la confrontation entre les vestiges archéologiques et l'art de leur époque, qui sépare radicalement peinture et sculpture. Les œuvres exhumées portent encore des traces de peinture, qui interpellent aussi bien J.-J. Winckelmann, le comte de Caylus que Quatremère de Quincy ${ }^{4}$.

Ce débat est nourri en filigrane par les avancées en physique effectuées par Isaac Newton ${ }^{5}$ sur la connaissance de la lumière, qui est blanche, et des couleurs: ce dernier met en évidence la composition de la lumière, comme la somme de toutes les couleurs perceptibles par l'œil humain, ce qui contredit l'expérience de l'artiste, qui, en mélangeant les pigments, n'obtient qu'un gris sale.

Les découvertes de sculptures antiques polychromes jalonnent les XVIII ${ }^{\mathrm{e}}$ et XIX ${ }^{\mathrm{e}}$ siècles: finalement acceptée pour

Axelle Davadie, conservateur C2RMF/Restauration/Filière sculpture, (axelle.davadie@culture.gouv.fr). Édouard Papet, conservateur en chef, musée d'Orsay (edouard.papet@musee-orsay.fr). Nathalie Pingaud, assistant-ingénieur de recherche, C2RMF (nathalie.pingaud@culture.gouv.fr). Anne-Solenn Le Hô, ingénieur de recherche, C2RMF (anne-solenn.le-ho@culture.gouv.fr). Agnès Cascio, restauratrice (agnes.cascio@free.fr). Juliette Levy-Hinstin, restauratrice (j.levyhinstin@gmail.com). Marie-Emmanuelle Meyohas, restauratrice (me.meyohas@gmail.com). 
l'art grec, cette polychromie continua de susciter un débat lorsqu'il s'agissait d'œuvres modernes, telle la Vénus de Gibson ${ }^{6}$, dont la réception fit naître une controverse violente. L'exposition à Dresde puis à Berlin ${ }^{7}$ organisée par Georg Treu, conservateur des collections de Dresde et archéologue à Olympie, est une invitation à la réflexion. Lors de sa conférence «Sollen wir unsere Statuen bemalen ${ }^{8}$ ? ", Treu abordait directement la polémique entre les défenseurs d'une statuaire moderne polychrome, à l'imitation de l'antique, et ceux qui la rejetaient. Les fouilles de Délos, menées par l'École française d'Athènes, livrèrent dès les années 1890 un grand nombre de sculptures polychromes; elles jouèrent ainsi un rôle important dans le progrès des connaissances ${ }^{9}$. Depuis les années 1960, la polychromie des sculptures antiques redevient un sujet d'étude, qui progresse en fonction de l'intérêt des savants et des avancées technologiques. Grâce à l'utilisation du vidéomicroscope, les examens de laboratoire et ceux faits sur place révèlent les traces infimes de cette polychromie et de sa mise en ouvre ${ }^{10}$.

J.-L. Gérôme, qui voyagea en Italie dès 1844, puis dans l'Empire ottoman en 1855 et en Égypte dès 1857, fut le chef de file du courant néo-grec et montra un intérêt constant pour les découvertes archéologiques et la question de la polychromie dans la statuaire grecque. Ce n'est donc pas un hasard s'il prit une part active à ce débat: ses œuvres à partir des années 1880 sont tout à la fois des essais et des manifestes. Sa production compte plusieurs marbres teintés et, semble-t-il, trois plâtres polychromes ${ }^{11}$.

Si la polychromie des sculptures antiques est assez bien documentée ${ }^{12}$ et ce, quel que soit le support, nous ne disposons plus pour le monde grec de sources textuelles originales de façon directe, tels les écrits théoriques des artistes grecs ${ }^{13}$.

On aimerait considérer cette œuvre ultime comme l'aboutissement des recherches de Gérôme dans la mise en couleurs de sculptures. Il faudrait pouvoir comparer les techniques employées sur les trois autres plâtres polychromes, ce qui, à ce jour, n'est pas possible. Qu'il s'agisse du plâtre préparatoire pour la Joueuse de cerceau ${ }^{14}$ (1891), de celui pour le buste de Bellone (1892), avec une garniture de strass ${ }^{15}$, ou encore de Sarah Bernhard $t^{16}$, aucune étude ne fut, à notre connaissance, menée dans cette perspective.

Dans un contexte créatif qui vit éclore d'autres essais, plus ou moins bien accueillis, que ce soient les sculptures de Charles Cordier ${ }^{17}$ ou l'Athéna Parthénos de Charles Simart (1855), les recherches de Gérôme prennent donc un relief particulier, d'autant que la découverte par un de ses anciens élèves, Osman Hamdi Bey ${ }^{18}$, d'un sarcophage polychrome en 1887, suscite le commentaire suivant de la part du sculpteur $^{19}$ : «Ressusciter la sculpture polychrome qui est essentiellement décorative.» Le caractère novateur de ces recherches est généré par l'époque et les découvertes archéologiques, mais la réduction de la polychromie à un rôle décoratif en détruit la portée. Il faut cependant noter qu'aucun autre artiste ne tenta de colorer les marbres à la cire, comme cela est précisé pour la Joueuse de boules ${ }^{20}$ (1902).

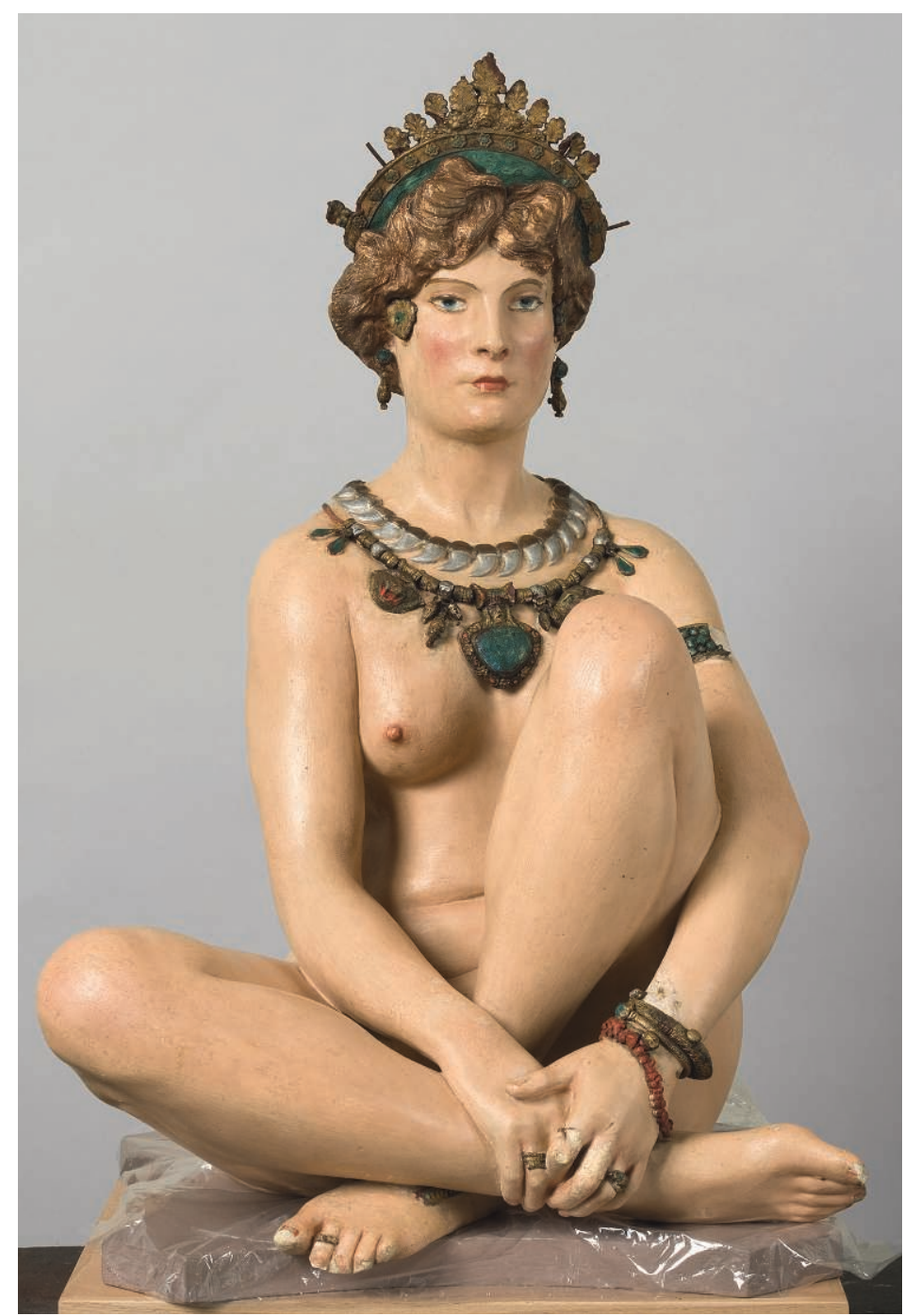

Fig. 1. La polychromie de la Corinthe apparaît saturée et salie lors de son entrée dans les collections du musée d'Orsay, H. 47,5 cm. (C) C2RMF/François Lauginie.

Il faut donc se résoudre à considérer, s'agissant de Corinthe, en l'absence d'archives explicites et d'études parallèles des autres plâtres polychromes, que l'inspiration est peut-être antique, mais la réalisation propre à Gérôme. Si les matériaux et techniques employés pour peindre ce plâtre diffèrent de ceux que les Grecs de l'Antiquité utilisaient pour leurs statues, on peut néanmoins mettre en évidence une similitude dans le traitement de la chevelure: peinte en brun-rouge comme sur les figurines de Tanagra, avec des reflets à la bronzine par Gérôme.

La Corinthe n'est pas une tentative isolée dans l'œuvre de Gérôme ni au XIX ${ }^{\mathrm{e}}$ siècle, même si la polychromie sur plâtre reste rare; inscrite dans son époque, cette œuvre fait-elle la synthèse entre la connaissance archéologique accessible au public, le goût bourgeois et la sensibilité de l'artiste ou n'estelle qu'un décalque de la polychromie antique considérée à cette époque comme une peinture à la cire? 


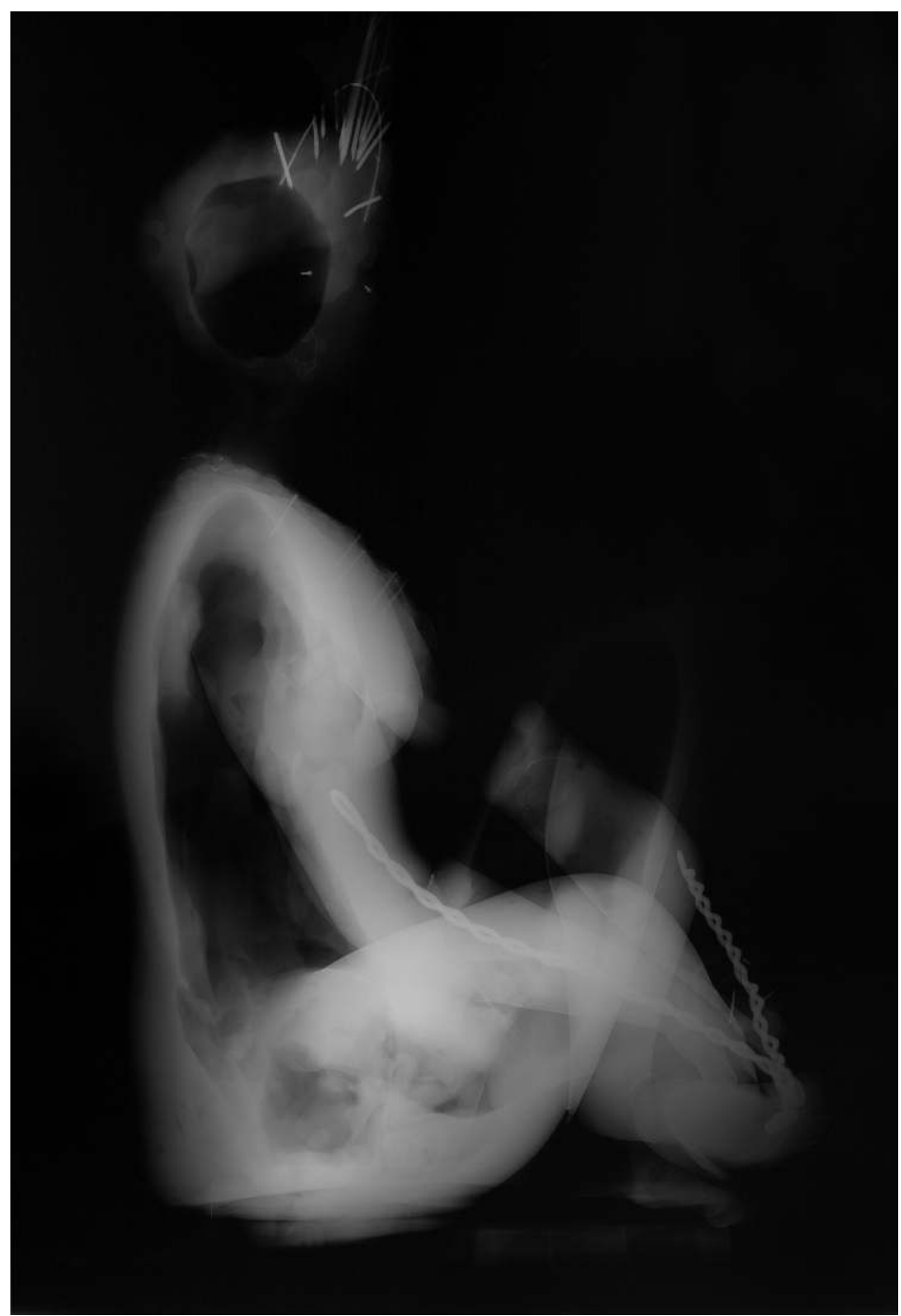

Fig. 2. Radiographie de l'œuvre.

(C) C2RMF/Thierry Borel et Jean Marsac.

\section{Lecture(s) de la Corinthe}

Corinthe $^{21}$ (fig. 1), dernière œuvre à laquelle Gérôme travaillait l'année de son décès, résume le parcours du sculpteur et en constitue en quelque sorte le testament artistique. Le modèle en plâtre polychrome, acquis en 2008 par le musée d'Orsay, est l'un des rares plâtres originaux de Gérôme qui subsiste; il a servi de modèle au marbre exposé de façon posthume au Salon des Artistes français de $1904^{22}$. Peint, luxueusement orné de bijoux en bronze doré et émaillé exécutés par le fondeur Hébrard et agrémentés de pierres semi-précieuses, ce marbre, aujourd'hui non localisé, aurait été terminé après la mort de Gérôme par Louis-Émile Decorchemont, qui était alors son assistant. Subsiste une version réduite, posée sur le chapiteau corinthien en bronze doré d'une colonne cannelée en marbre vert. Les factures d'Hébrard pour les bijoux du marbre permettent vraisemblablement de dater le plâtre de 1903, année au cours de laquelle la majeure partie d'entre eux ont été réalisés. Corinthe fut par la suite diffusée par la maison Siot-Decauville sous forme d'éditions légèrement plus petites en bronze doré, rehaussées de pierres semi-précieuses, sans colonne, le chapiteau seul émergeant "archéologiquement» d'une terrasse rocheuse. Comme Tanagra, Corinthe joue, en bien plus sulfureux, sur l'idée grecque de la Tyché, mais une fois de plus, Gérôme convoque l'Orient pour évoquer l'érotisme antique. Puissante cité du Péloponnèse au commerce florissant avec l'Orient, l'attrait majeur de Corinthe sur l'imaginaire du XIX ${ }^{\mathrm{e}}$ siècle résidait dans le souvenir des hiérodules (prostituées sacrées), cantonnées dans l'enceinte du temple consacré à la déesse Aphrodite. L'inscription latine «NON LICET OMNIBUS/ADIRE CORINTHUM » sur le cartouche de la version définitive de l'œuvre en marbre évoque un adage antique rapporté par le géographe Strabon: «Les riches marchands, les militaires venaient s'y ruiner et s'y ruiner irrémédiablement, ce qui a donné lieu à ce proverbe bien connu: "tout homme n'est pas fait pour se rendre à Corinthe ${ }^{23}$ ". " Le lien avec l'Orient est peut-être à rechercher du côté de Babylone où les hiérodules locales, selon Hérodote, se tenaient «assises dans l'enceinte sacrée d'Aphrodite avec une couronne de corde autour de la tête. [...] Les étrangers y circulent et font leur choix. Lorsqu'une femme a pris place en ce lieu, elle ne retourne pas chez elle avant qu'un étranger lui ait jeté de l'argent sur les genoux et qu'elle se soit unie à lui à l'intérieur ${ }^{24}$ ». Néanmoins, il demeure encore aujourd'hui difficile de savoir exactement pourquoi Gérôme décida, à la fin de sa vie, de représenter une allégorie de Corinthe, même si cette figure, assise au sommet d'une colonne, évoque d'une manière très irrévérencieuse l'iconographie des saints stylites des débuts du christianisme à laquelle Gérôme a travaillé ${ }^{25}$. Par ailleurs, avec Corinthe, le sculpteur évoque également une cité qui était l'objet de nombreux récits légendaires sur l'invention artistique, passant pour avoir vu naître la peinture, l'art du portrait sculpté et le chapiteau éponyme ${ }^{26}$ : c'est bien sur l'un d'eux, très proche d'un chapiteau trouvé à Épidaure en 1884, et considéré alors comme le prototype même du chapiteau corinthien, qu'est installée la courtisane. Comme pour les Gladiateurs ${ }^{27}$ vingt-cinq ans plus tôt, Gérôme s'est documenté assez solidement pour donner de la vraisemblance à cette figure de fantaisie. Les bijoux doivent tout à ceux, grecs et étrusques, de la collection Campana, entrée au Louvre en 1863, telle la bague visible sur l'œuvre définitive ${ }^{28}$, ainsi qu'à un ouvrage par Eugène Fontenay, Les Bijoux Anciens et Modernes, publié en 1887 et abondamment illustré. Comme souvent chez Gérôme, ce grand souci d'exactitude dans les sources d'inspiration va de pair avec l'anachronisme: Corinthe est coiffée comme une Parisienne du début du Xx $\mathrm{X}^{\mathrm{e}}$ siècle. Il n'est pas non plus impossible que Gérôme se soit enfin inspiré d'une œuvre très célèbre au XIX ${ }^{\mathrm{e}}$ siècle, le Buste d'Elche, découvert en Espagne en 1897, paré de bijoux, et considéré alors comme le parfait exemple de la complexe synthèse des civilisations méditerranéennes. Dernière mise en scène savante de Gérôme, variation hyperréaliste de la femme fatale, du sphinx énigmatique, Corinthe est avant tout une sculpture crue, troublante, un «Cabinet de curiosités» régi par la confusion des genres et assemblé par Gérôme sous couvert d'un historicisme rigoureux, Corinthe, que l'on peut également 
lire comme une allégorie du demi-monde parisien, constitue sans doute l'un des plus spectaculaires nus post-symbolistes conçus au XIX ${ }^{\mathrm{e}}$ siècle, exsudant une sexualité morbide. L'«idole indienne ${ }^{29}$ » de Gérôme reçut un accueil mitigé, plutôt défavorable: «Autour d'elle se groupe un public absolument ahuri. Gérôme a-t-il voulu discréditer à tout jamais la statuaire polychrome qu'affectionnaient les Grecs? [...] Ce n'est pas eux qui eussent peint ces chairs aux tons fades comme la cire des poupées de coiffeurs ${ }^{30}$. » Seul le critique Tristan Lecière considéra que «trois œuvres dominent la sculpture aux salons de 1904: le Penseurde Rodin, le Mineur de Constantin Meunier et la Corinthe de Gérôme ${ }^{31}$ » et fournit une analyse élogieuse des recherches de Gérôme sur la polychromie illusionniste, tout en y décelant bien l'aporie: "Je ne crois pas qu'on ait jamais cherché à s'approcher aussi près du réel que Gérôme [...]. Cette Corinthe est un chef-d'œuvre, mais le chef-d'œuvre de la fin d'un art: elle n'ouvre pas la route à des essors nouveaux; elle marque un aboutissement; elle est le dernier terme de la sculpture polychrome. On pourra bien entendu trouver encore des combinaisons imprévues de polychromies, des arrangements inédits de matières diverses; on n’ira guère plus loin ; c'est une forme d'art qui a touché à sa limite. ${ }^{32}{ }^{\text {» }}$

\section{Étude de l'œuvre}

\section{Mise en ouvre}

D'une remarquable exactitude morphologique ${ }^{33}$, le corps de la Corinthe traduit la volonté marquée d'imiter la nature. Le sculpteur a, dans un premier temps, modelé la figure en terre, puis il l'a moulée. Il est possible qu'à partir de cette première épreuve originale, des surmoulages, agrandissements ou réductions aient été effectués, en plâtre également, destinés notamment à la réalisation des modèles en bronze ou en marbre. Ces modèles de fonderie ou de mise au point ${ }^{34}$ ont disparu. En revanche, la Corinthe du musée d'Orsay (fig. 1), travaillée avec soin, est un modèle en plâtre probablement destiné à être conservé dans l'atelier de l'artiste. Dans ce sens, il s'agit vraisemblablement du plâtre original, orné de bijoux et peint par l'artiste, probablement le projet pour la version en marbre, qui est de dimensions légèrement supérieures et également peinte. La sculpture est le fruit du travail d'un praticien très expérimenté. L'épreuve est issue d'un moule à pièces, également appelé «moule à bon creux». Si les traces de coutures ont été, pour la plupart, soigneusement éliminées à la sortie du moule lors de l'étape de la reparure, certaines sont encore visibles.

Le corps de la Corinthe est offert au regard sous tous ses angles: sa position dans l'espace n'est pas, sur le plan technique, simple à mouler. Les contre-dépouilles sont nombreuses. Il n'est donc pas surprenant que le moulage de l'œuvre ait comporté des abattis: la disposition des vides dans le plâtre suggère que la tête et le buste ont été moulés à part, ce qui pourrait également être le cas de la jambe gauche. L'ensemble de ces pièces en plâtre aurait été assemblé au «socle» de la figure, constitué de la partie inférieure du bassin, des bras, mains et jambe droite repliée, qui forment un bloc à l'intérieur duquel l'armature métallique a été placée lors du moulage.

\section{Examens et analyses}

La radiographie, effectuée par Thierry Borel ${ }^{35}$, apporte des éléments d'information à la fois sur l'exécution du moulage en plâtre et sur le mode de fixation des bijoux en cire, retenus par des pointes aisément repérables (fig. 2). Le modèle en plâtre est partiellement creux. Les cavités, plus sombres sur le cliché, sont visibles dans la tête, le buste, ainsi que dans les bras et les cuisses. La paroi du tirage en plâtre est relativement peu épaisse, donc fragile. De manière à renforcer les parties les plus vulnérables (bras et jambes), plus exposées aux chocs, une armature a été insérée dans le moule. Constituée d'un double fil torsadé en métal ferreux ou cuivreux, elle est formée de trois branches disposées en Y: l'une prend naissance dans la zone située juste au-dessus du coude droit, et rejoint la seconde, qui se poursuit jusqu'au coude gauche, tandis qu'une troisième branche se prolonge dans le pied droit. Le «nœud» de ce renfort métallique est situé à l'intérieur des mains jointes sur la cheville droite.

L'observation de la surface du matériau des bijoux, travaillée avec une extrême précision, indique que les éléments ont été obtenus par moulage. Le fait que ceux-ci soient montés sur un fil d'argent de même que la couleur rouge suggèrent le rapprochement avec le travail d'un orfèvre.

Fils d'orfèvre, Gérôme est familier du métier, connaît les matériaux précieux et s'intéresse vivement aux bijoux archéologiques, reproduits et miniaturisés sur la Corinthe, dans un esprit d'imitation illusionniste.

La prise d'empreinte des bijoux a été effectuée selon différents procédés. Certains ornements ont été moulés en deux parties assemblées, telles les colombes des boucles d'oreilles, le fil d'argent ayant été mis en place lors de la réunion des deux coques (fig. 3); d'autres éléments de la parure ne comportent qu'une face moulée, comme les palmettes surmontant le diadème.

Avant toute prise de décision, des analyses ont été réalisées. L'étude de la polychromie a pour objectif de mieux connaître la confection générale d'une ouvre peinte, en se fondant sur la caractérisation des techniques picturales, tout comme des constituants utilisés par l'artiste, et d'apporter ainsi des informations indispensables aux opérations de restauration. En sculpture, cette démarche nécessite souvent la prise d'échantillons, seul moyen, aujourd'hui, qui permette de mettre en évidence de façon précise et exhaustive la chronologie des différentes mises en couleur. Ces échantillons extrêmement petits sont préparés sous forme de coupes stratigraphiques qui pourront être observées et analysées ${ }^{36}$, puis conservées de façon pérenne.

Du point de vue de la polychromie ${ }^{37}$, la statue est essentiellement constituée de «carnations » et d'ornements (collier, bagues, bracelets, etc.). L'étude, effectuée à partir de dix 
Fig. 3. La Corinthe est parée de bijoux archéologiquement exacts, œuvres conçues et réalisées par J.-L. Gérôme à la suite de ses lectures et documentations. (c) C2RMF/Anne Chauvet.

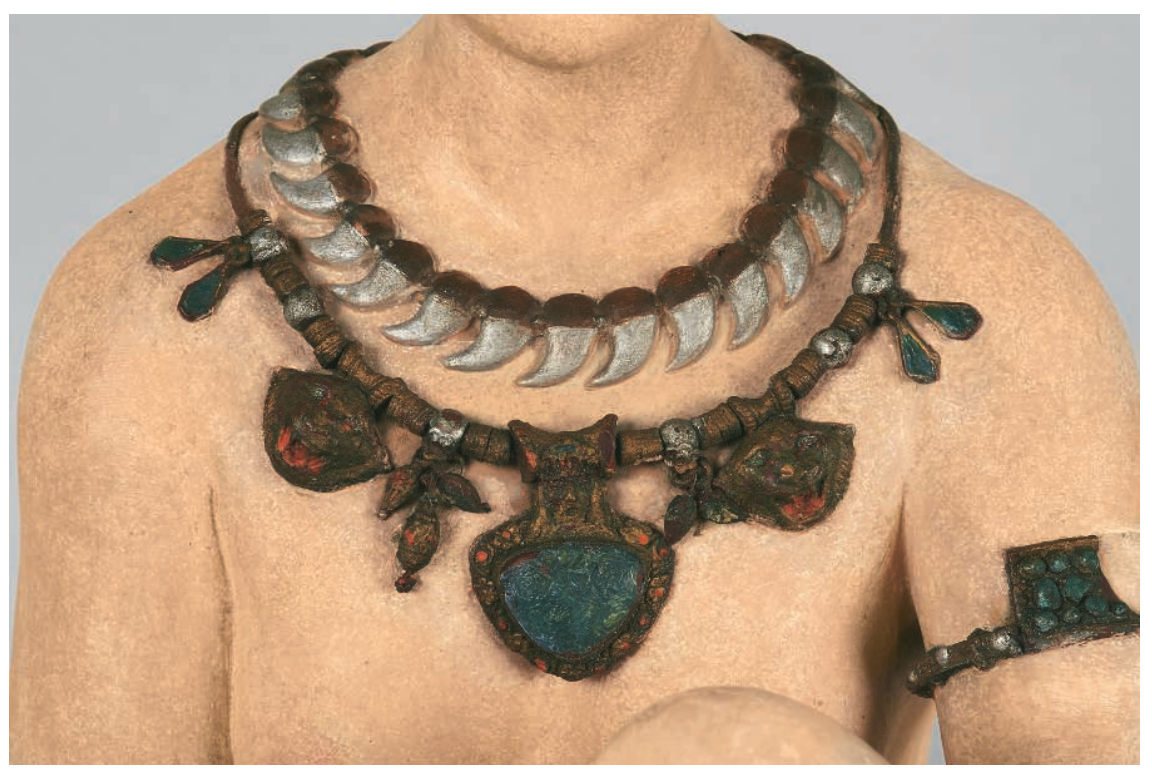

échantillons, a été axée sur la composition des couches picturales, et, pour les parures, sur leur mode d'exécution.

Concernant les carnations, quatre échantillons ont été prélevés au niveau des orteils senestres et dextres, ainsi que sous la fesse senestre de la Corinthe. Les coupes obtenues (fig. 4) ont révélé une stratigraphie constituée du support en plâtre revêtu de deux couches de carnation : la polychromie originale et un repeint.

Gérôme a peint les carnations sur la totalité de la sculpture. Finement craquelée, la couche de peinture est si peu épaisse qu'elle laisse parfois transparaître la blancheur du plâtre sous-jacent. Le ton est un rose très pâle, presque nacré, qui rappelle les chairs des peintures sur toile de l'artiste. Sur l'épiderme du plâtre, il n'y a pas de préparation blanche, mais uniquement une fine couche d'encollage. La finesse de la couche picturale laisse voir les stries de lissage du plâtre, et les traces de pinceau, rapidement brossées, imitent délibérément et habilement le léger relief du grain de la peau.
La couche picturale initiale a été appliquée à l'aide de grains de bleu céruléum (orthostannate de cobalt II) et de laque rouge dans une matrice de blanc de plomb et de blanc de zinc. Cette première couche, observée également sur le collier en dents de sanglier figuré en relief et sur la chevelure de la Corinthe, montre qu'elle a été appliquée en totalité sur l'œuvre, faisant également fonction de couche de préparation.

Le repeint est composé d'un mélange d'ocre rouge, de blanc de zinc et de kaolinite.

L'analyse du liant par infrarouge à transformée de Fourier a révélé la présence d'huile (fig. 5). Néanmoins, il est impossible de déterminer avec précision si le liant analysé appartient à la polychromie originale, au repeint ou bien aux deux. Au niveau des orteils, où des zones lacunaires étaient présentes, des retouches au blanc de titane additionné d'ocre et de pigment au cadmium ont été appliquées ponctuellement pour atténuer les manques des parties écaillées.

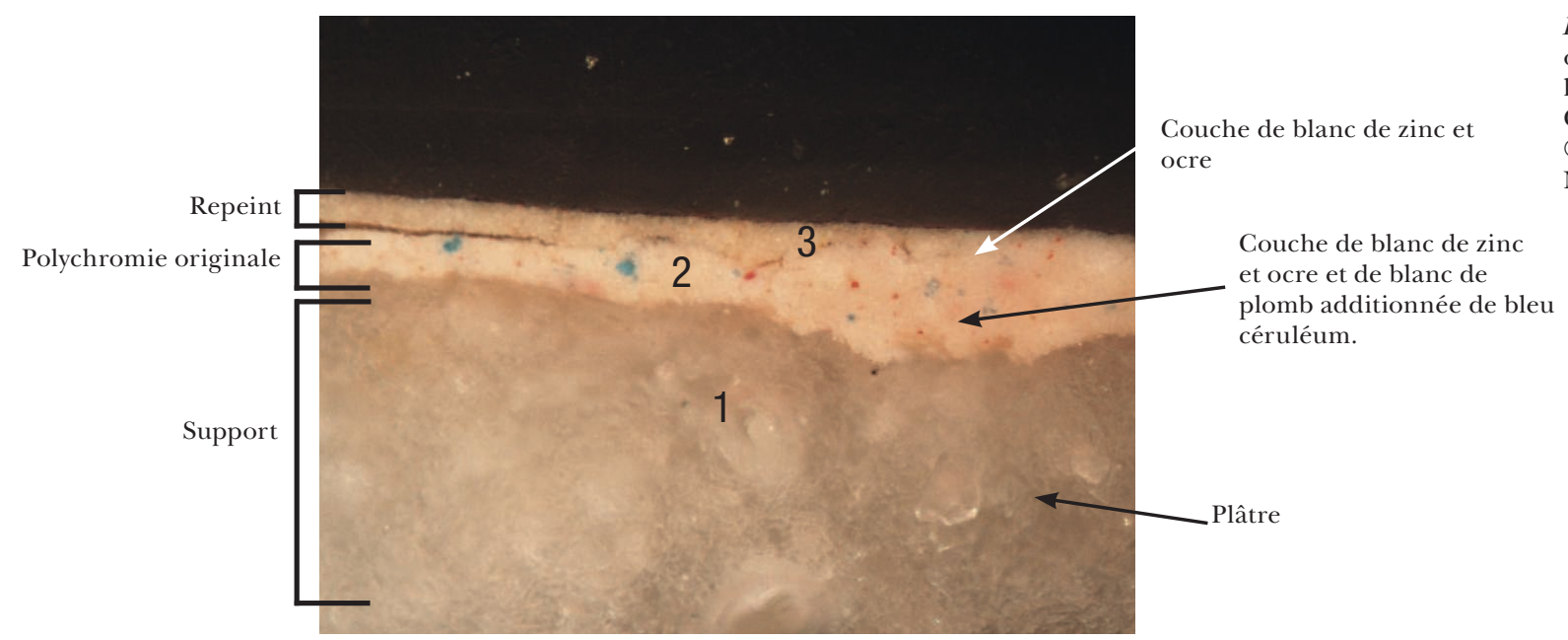

Fig. 4. Microscope optique en lumière blanche. Carnation. (c) C2RMF/ Nathalie Pingaud. 


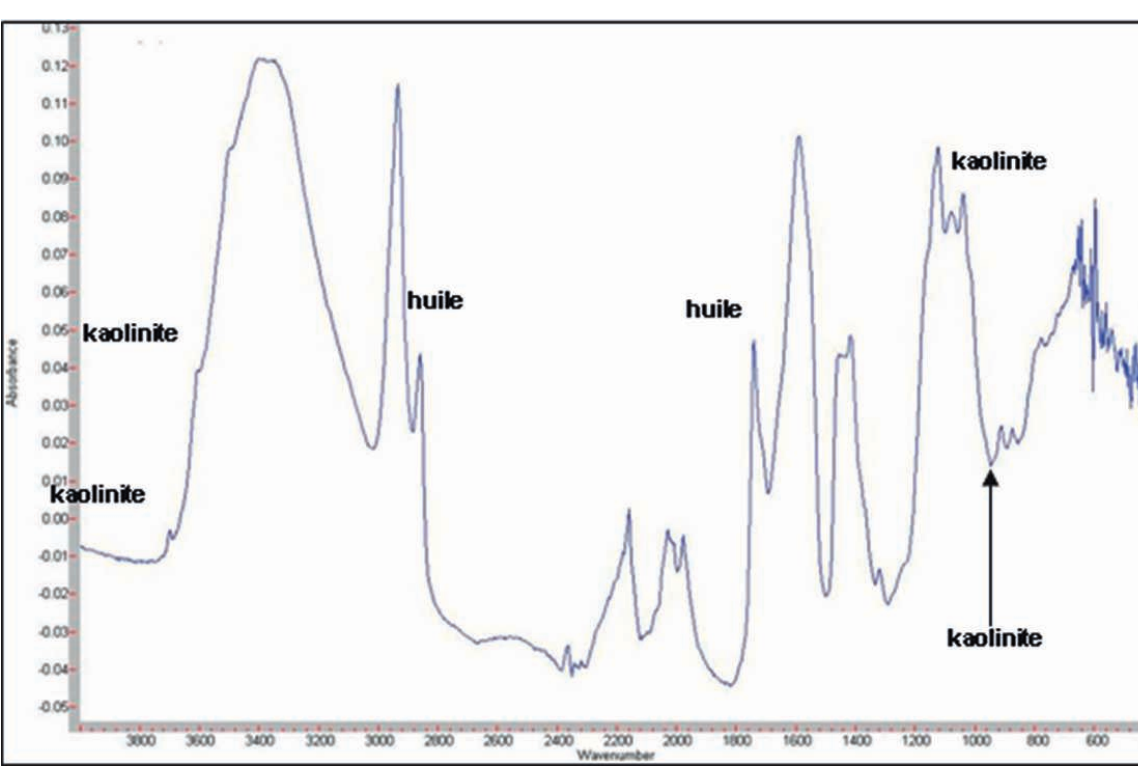

Fig. 5. Spectre IRTF d'un

micro-échantillon collecté

en transmission avec une

cellule diamant (microscope

Digilab Excalibur FTS

4000/UMA 600-64 scans-

résolution $\left.4 \mathrm{~cm}^{-1}\right)$.

(c) C2RMF/Anne-Solenn

Le Hô.

Les yeux sont d'un gris violacé subtil, soulignés d'une ligne noire qui se prolonge vers les tempes. La bouche est d'un rose assez pâle. Les sourcils originaux sont d'un brun très clair, placés bien plus haut que ceux du premier repeint, lesquels étaient visibles avant restauration.

Après l'élimination du repeint, la chevelure est apparue d'un brun-roux translucide, appliqué en couche assez fine où s'entrecroisent les traces de brosse. Ce brun-roux nuancé (mélange d'ocre, de blanc de zinc et de blanc de plomb), appliqué sur la couche de carnations, est rehaussé de mèches dorées à la «bronzine», dont le reflet métallique, semblable à celui des bijoux, accentue le caractère surnaturel et précieux de l'idole. Il semble que deux nuances de «bronzine», plus ou moins chargée de poudre de cuivre, aient été appliquées sur les cheveux.

En ce qui concerne les ornements (fig. 6a et 6b), deux matériaux ont été mis en œuvre: le matériau plâtre pour le collier en dents de sanglier d'apparence argentée, et un mélange de cire et d'amidon pour le bracelet de cheville (symbole de prostitution en Égypte ancienne ${ }^{38}$ ). Bien que non analysés, les autres bijoux (bagues, bracelets de bras, boucles d'oreilles et collier à médaillon) paraissent être de même facture que le bracelet de cheville. La couche argentée du collier a été obtenue par application d'une feuille d'aluminium, posée sur la couche de «carnation» qui, ici, a joué le rôle d'une couche de préparation. La polychromie du bracelet de cheville a consisté en une application de bronzine (alliage de cuivre et de zinc en paillettes).

Sur les bijoux, la polychromie originale, qui n’a été que très ponctuellement reprise, est en grande partie visible aujourd'hui. Elle semble avoir été exécutée très librement. La cire rouge est revêtue d'une «bronzine», rehaussée de touches de couleur: rouge vif sur les gueules des lionnes, le bracelet de corail, les perles des sandales; bleu-vert évoquant

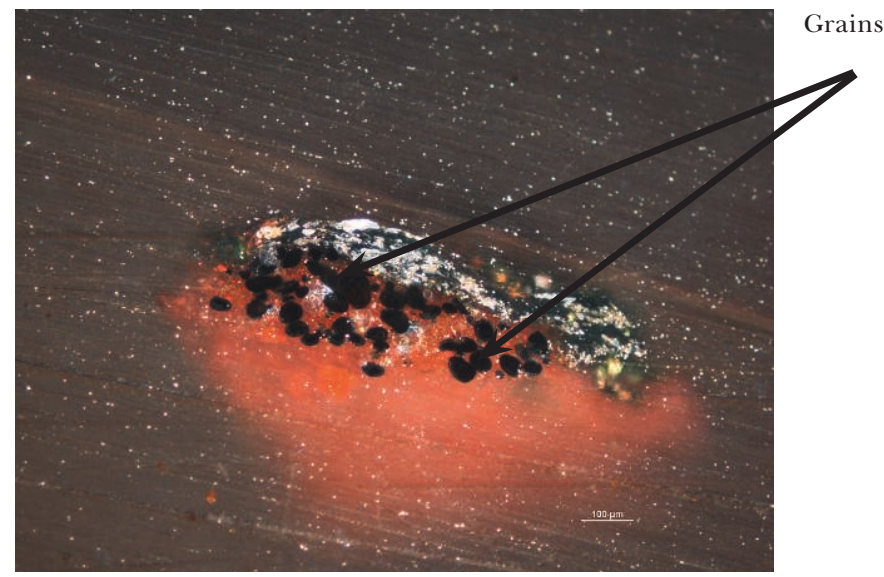

Fig. 6 a. Microscope optique en lumière blanche. Chaîne de cheville: test au Lugol. (c) C2RMF/Nathalie Pingaud.

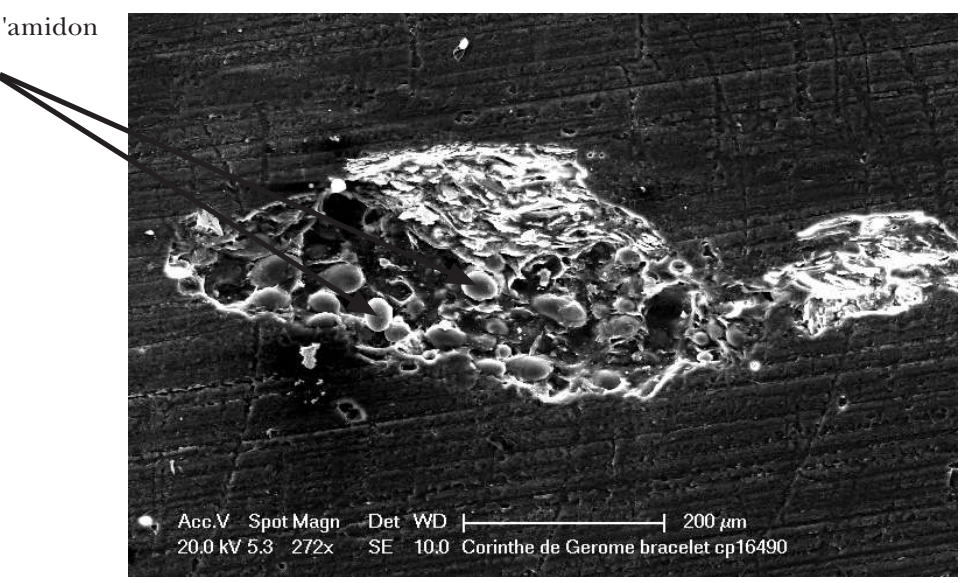

Fig. 6 b. Image MEB-SE de la stratigraphie de la chaîne de cheville dévoilant les grains d'amidon dans la matrice de cire. (c) C2RMF/Nathalie Pingaud. 
une pierre de lapis-lazuli un peu veiné ou peut-être une turquoise. Ce bleu profond revêt le plat du diadème et le pectoral central du grand collier (mélange d'arséniate de cuivre ou vert de Véronèse et de bleu de Prusse, chargé de blanc de sulfate de baryum). Les dents de sanglier, montées sur le collier, évoquent le casque «primitif» mycénien et mettent en lumière le jeu d'emprunts archéologiques effectués par l'artiste.

\section{Enjeux et choix de la restauration}

Avant restauration, la polychromie originale était presque entièrement dissimulée sous un repeint unique, d'époque moderne. Ce repeint avait été suivi, plus récemment, de retouches apposées lors de différentes campagnes, dissimulant des accidents sur l'ensemble de la surface. Les bijoux n'étaient que faiblement retouchés, mais leur éclat était terni par l'oxydation de la «bronzine» qui les recouvrait.

\section{Ordre d'exécution de la polychromie}

L'observation de la surface peinte sous loupe binoculaire a permis de comprendre l'ordre des différentes étapes d'application de la polychromie sur le plâtre et la cire des bijoux. Gérôme a commencé par les carnations, qu'il a peintes en tout premier lieu sur l'ensemble de la surface, y compris les cheveux. Après avoir revêtu la sculpture d'une couche de carnation, il a peint les traits du visage, yeux, sourcils, bouche et la chevelure. Ensuite, les bijoux en cire rouge ont été placés sur le corps: accrochage des boucles d'oreilles et des pendants de tempe, collage et fixation dans le plâtre, au moyen de pointes métalliques, des bracelets, du collier, des bagues aux mains et aux pieds, des sandales; sur d'autres pointes préalablement fichées sur la sculpture, le diadème a été façonné, avant d'être peint, tout comme les autres bijoux, à la bronzine. Lors de la dernière étape de mise en couleur des bijoux, la chevelure a été également rehaussée de touches de bronzine. Enfin, la peinture bleu-vert imitant les pierres serties dans les bijoux correspond à la dernière touche de couleur (fig. 4).

\section{Le premier repeint}

Vraisemblablement exécuté par un peintre ou un amateur averti, le premier repeint a été réalisé directement sur la polychromie originale, peut-être parce que les carnations, salies, étaient devenues un peu grises (fig. 1). Le corps a alors été peint dans un rose orangé, opaque, appliqué en épaisseur. Le visage est devenu vivement coloré, presque maquillé. Les yeux, au regard fixe et un peu vide, ont été peints de touches d'un bleu vif, ponctué d'accents blancs, et les paupières ont été soulignées d'une épaisse ligne brune et noire. La bouche a été colorée de rouge carmin, et les sourcils d'une fine ligne courbe brune très régulière. Le style de ce repeint pourrait le situer après les années 1930, ce que semble confirmer une photographie ancienne ${ }^{39}$.

Ce repeint a été suivi plus récemment de quelques retouches colorées destinées à masquer les lacunes de polychromie mettant à nu le plâtre. Puis il a ensuite été retouché pour masquer les lacunes (voir plus haut).

\section{État de conservation}

Du fait de son caractère composite, et en particulier de l'emploi de cire et de métal appliqués à même le plâtre peint, l'œuvre présente une grande vulnérabilité. Dans l'ensemble, l'état du plâtre est satisfaisant sur le plan structurel. Sous l'effet de petits chocs, quelques petits enfoncements se sont produits sur l'ensemble du corps, dont certains ont été comblés avant l'application du premier repeint.

L'épiderme de l'œuvre présentait un empoussièrement général et des salissures. Sur les bijoux en cire, de rares soulèvements de polychromie étaient visibles, notamment sur l'élément central du pectoral. La structure composite des bijoux nuit à leur stabilité et à leur conservation. Or, les bijoux de cire $^{40}$ (une cire avec de l'amidon est d'autant plus vulnérable aux variations hygroscopiques) sont montés sur du métal: les boucles d'oreilles et les pendants de tempe sur un fil d'argent très souple, tandis que sur le diadème, la cire est appliquée sur des tiges de cuivre plus épaisses servant d'armature. Beaucoup sont en partie perdus, comme le montre la comparaison avec la version de Corinthe en marbre, tels les palmettes ou des éléments du diadème, ou encore les parties libres des pendants de tempes et les sandales. Ils constituent, d'une manière générale, les points de fragilité les plus extrêmes de l'œuvre.

\section{Traitement de restauration}

À la suite de l'étude de l'œuvre et de sa polychromie, des mesures de conservation préventive ont été menées dans un premier temps. Le refixage de la polychromie localement soulevée, dans les bijoux et les carnations, a été effectué, parallèlement au dépoussiérage de l'ensemble.

L'étude avait révélé que sous le repeint, la polychromie originale semblait bien conservée, aussi bien sur la chevelure que sur le corps et le visage. Retrouver la peinture de Gérôme sur l'une de ses sculptures en plâtre représentait un enjeu et un défi exceptionnels. Cependant, les tests préalables avaient montré que l'intervention était extrêmement délicate. En effet, le liant du repeint est très semblable à celui des carnations originales et l'adhérence entre ces deux couches est forte: pour cette raison, un dégagement chimique n'était pas envisageable. L'intervention longue et difficile à mener devait donc, dans son intégralité, être réalisée au scalpel, sous loupe binoculaire (grossissement 3 à 5 selon les zones).

Lopération a donc été conduite pendant plusieurs mois. Progressivement, les pâles carnations du corps peint par 


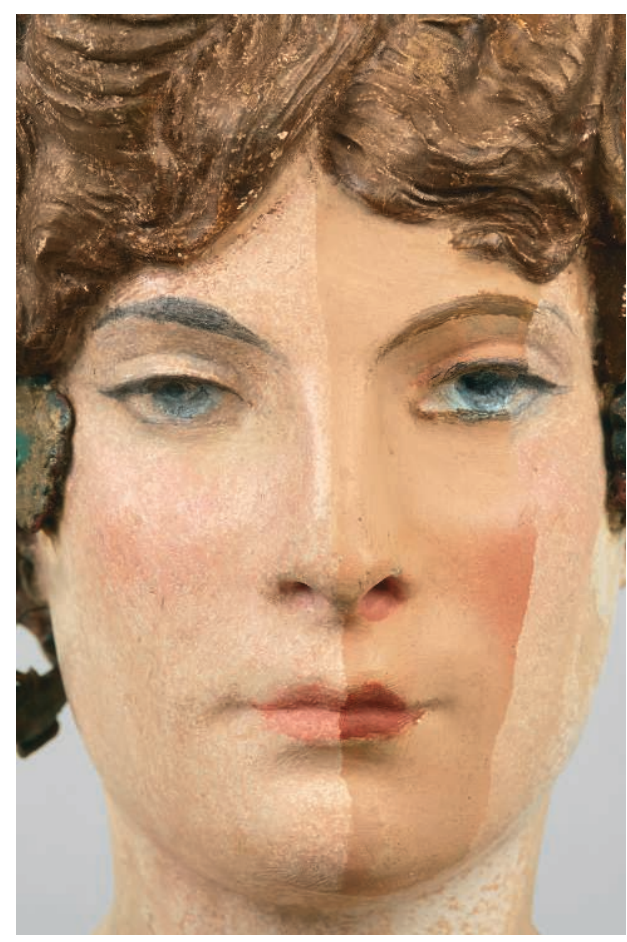

Fig. 7. Dégagement de la polychromie originale achevé sur le côté gauche et en cours sur le côté droit: le regard retrouve son expression originelle.

(C) C2RMF/Anne

Chauvet.

Fig. 8. Après restauration, la Corinthe révèle sa polychromie originale. (C) $\mathrm{C} 2 \mathrm{RMF} / \mathrm{Ann}$ e Chauvet.

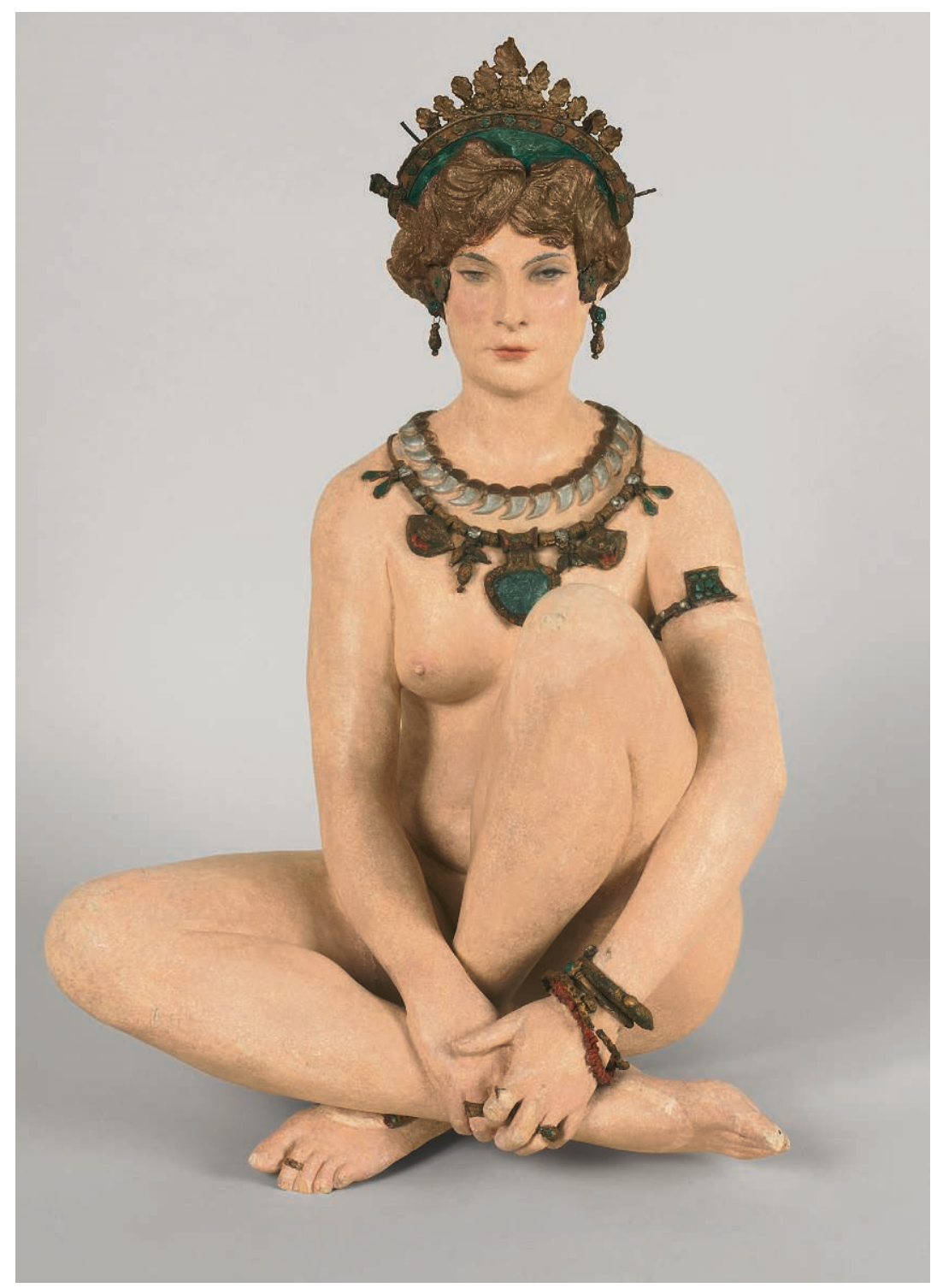

Ainsi la sculpture (fig. 8) a-t-elle retrouvé une esthétique

Gérôme sont réapparues, ainsi que les nuances subtiles du modelé du corps, que le repeint avait alourdies. Sur le visage, l'intervention a été plus décisive encore, la bouche et les yeux retrouvant leur expression originelle, en accord avec la position dominante de la courtisane, du haut de sa colonne (fig. 7). Enfin, pour documenter l'intervention, des témoins du repeint ont été volontairement conservés dans les zones inaccessibles au regard.

Dans les lacunes de la polychromie, notamment les genoux, plus exposés, apparaissait le plâtre blanc. Des retouches colorées ont donc été pratiquées pour redonner son unité à la surface peinte. De façon à être décelées par un œil averti, elles sont d'un ton légèrement plus clair que celui des carnations originales.

Sur la chevelure, le dégagement de la polychromie originale a pu être mené par voie chimique, avec des solvants tels l'éthanol. Les bijoux, peu repeints, ont simplement été minutieusement nettoyés. et un sens qui l'inscrivent profondément à la fois dans le XIX ${ }^{\mathrm{e}}$ siècle et dans l'œuvre de Gérôme.

La polychromie de ce plâtre révèle les choix esthétiques de l'artiste: des carnations très légères, une chevelure flamboyante, des bijoux colorés. En l'absence de strict équivalent antique (plâtre peint), il est délicat de trancher le débat: imitation des techniques anciennes ou libre interprétation par Gérôme? Nous pouvons cependant constater que la peinture utilisée est liée à l'huile sur le plâtre et qu'aucune cire n'entre dans la composition des carnations ou de la chevelure, à la différence de certains marbres. Il serait donc souhaitable que des analyses soient pratiquées sur les marbres et les plâtres polychromes afin de documenter plus finement les techniques et choix de l'artiste. 


\section{Notes}

Nous remercions Guy Bazile et Jean Marsac pour leur collaboration technique dans le montage des radiographies.

1. Gaborit J.-R., Ligot J., 1987.

2. Daumier, Les célébrités du juste milieu, musée d'Orsay, Paris.

3. Blühm A., 1996.

4. Polychromie, terme créé par A. Quatremère et frontispice de l'édition, Le Jupiter olympien ou l'Art de la sculpture antique, Paris, 1814 : reconstitution polychromée.

5. Prater A., 2002, p. 23-36.

6. Ostergaärd S. J., 2008, p. 78-105.

7. 1885, Dresden Staatlichen

Kunstsammlungen, Albertinum; Berlin Königliche Nationalgalerie.

8. "Devons-nous peindre nos statues?", conférence tenue au Königliches Polytechnikum.

9. Bourgeois B. et Jockey Ph., 2007 , p. 163-164.

10. Bourgeois B., 2007, p. 168, dont le rôle pionnier doit être salué; Brinkmann V. (éd.), 2010.

11. Ackermann G. M., 2000

12. Primavesi O., 2008, p. 24-77,

Østergaärd S. J., 2008, p. 78-105, Brinkmann V. et alii, 2008, p. 188-217.

13. Pline l'Ancien, Histoire naturelle,

XXXV, 36, Antigone, Xénocrate, Apelle.

14. Ackermann G., 2000, S 21, p. 388.

15. Ackermann G., 2000, S 27, p. 390.

16. Ackermann G., 2000, S 33, p. 392.

17. L'Arabe (bronze et onyx, Orsay

RF3598), Le Nègre du Soudan (bronze et onyx, Orsay RF2997), la Juive d'Alger (bronze émaillé, onyx et porphyre), par exemple.

18. Sarcophage dit d'Alexandre.

19. H. Metzger, 1990, p. 86-87.

20. Ackermann, 2000, S 57, p. 400, «un marbre frotté à la cire».

21. Ce paragraphe reprend notre étude: Papet É., 2009, p. 73-84.

22. Papet E, 2010, p. 329.

23. Strabon, Géographie, XII 3, 36.

24. Hérodote, Histoires, I, 199

25. Des Cars S., 2010, p. 143, cat. 83.

26. Pline l'Ancien, Histoire naturelle,

XXXV, 5, 43 .

27. Papet É., 2010, p. 131-133.

28. Papet É., 2010, cat. 193, p. 329.

29. Dieulafoy J., 1904, p. 481-482.

30. Dieulafoy J., 1904, p. 481-482.

31. Leclère T., 1904

32. Leclère T, 1904

33. Papet É., 2009. Photographie prise dans le vestibule de l'appartement de la famille Gérôme en 1931.

34. Les modèles de fonderie seraient recouverts de gomme laque et les modèles de mise aux points seraient munis de clous indiquant les chefs-points et portant la marque des points justes.

35. Radiographies exécutées par Thierry Borel, ingénieur au C2RMF, décédé en 2012. Nous aimerions lui dédier cet article.

36. Les observations détaillées ont été réalisées au microscope optique en lumière blanche et sous lumière ultra-violette. Les analyses élémentaires ont été effectuées à l'aide du MEB-EDS (Philips XL30 ESEM, couplé à un spectromètre de rayons $\mathrm{X}$ à dispersion d'énergie (système de détection SiLi) avec une tension d'accélération de $20 \mathrm{keV}$ ). Les analyses moléculaires ont été réalisées sur un spectroscope IRTF PerkinElmer Spectrum 2000. Les spectres infrarouges ont été obtenus par transmission à l'aide d'une cellule diamant de compression. Le signal a été mesuré sur une gamme spectrale allant de 4000 à $400 \mathrm{~cm}^{-1}$ avec une résolution spectrale de $4 \mathrm{~cm}^{-1}$ et une accumulation de 10 scans.

37. Pingaud, Le Hô, Étude de la

polychromie, dossier C2RMF 68501, rapport $\mathrm{n}^{\circ} 17840$

38. Elizabeth Abbott, 2003, p. 87.

39. Photographie récemment retrouvée par É. Papet.

40. Pingaud N., Le-Hô A.-S., op. cit. n. xxxvii.

\section{Bibliographie}

Abbott Elizabeth, 2003, trad. Paule Pierre, Histoire universelle de la chasteté et du célibat, Fides.

Ackermann G. M., 2000, Jean-Léon Gérôme, ACR, Courbevoie.

Bourgeois B., Jockey Ph., 2007, «Le marbre, l'or et la couleur. Nouveaux regards sur la polychromie de la sculpture hellénistique de Délos», dans Descamps-Lequime (dir.), 2007 p. $163-164$

Blühm A., 1996, The Colour of Sculpture, catalogue d'exposition Van Gogh Museum, 26 juillet-17 novembre 1996 , Amsterdam.

Brinkmann V., Primavesi O., Hollein M. (éds.), 2010, Circumlitio. The polychromy of antique and medieval sculpture, Proceedings of the J. D. Passavant Colloquium, Liebigshaus Skulpturensammlung, 10-12 December 2008 (Frankfurt, 2008), Hirmer Publishers, Münich.

Papet É., 2005, Daumier et les célébrités du juste milieu, BNP Paribas, Paris.
Descamps-Lequime S. (dir.), 2007, Peinture et couleur dans le monde grec antique, Actes du colloque organisé au musée du Louvre (10 et 27 mars 2007), musée du Louvre-Milan, Paris.

Des Cars L. (dir), 2010, Jean-Léon Gérôme (1824-1904), l'histoire en spectacle, SkiraFlammarion, Paris

Dieulafoy J., 1904, «Les Salons de 1904le Salon des Artistes français », Le Correspondant, avril 1904.

Gaborit J.-R., Ligot J. (dir.), 1987, Sculptures en cire de l'Égypte ancienne à l'art abstrait, RMN, Paris.

Hérodote, Histoires, 1993, I, Clio, CUF, trad. Ph. E. Legrand, Les Belles Lettres, Paris.

Leclère T., 1904, «La Sculpture», Salon, Paris, 1904, préface de Gustave Geffroy, Paris

Metzger H., 1990, «La correspondance passive d'Osman Hamdi Bey ", Mémoires de l'Académie des Inscriptions et Belles Lettres, n. s. XI, Paris, p. 86-87.

Østergaärd J. S., "The Polychromy of Antique Sculpture. A Challenge to Western Ideals" dans Circumlitio, p. 78-105.

Papet É., "Autour de la Corinthe de JeanLéon Gérôme", dans La Revue des Musées de France, Revue du Louvre, 2009-4, p. 73-84.

Pausanias, 2002, Description de la Grèce, VI, XVIII, 7, CUF, trad. J. Pouilloux, M. Casewitz, et A. Jacquemin, Les Belles Lettres, Paris.

Pline l'Ancien, 2003, Histoire naturelle, XXXV, CUF, trad. J.-M. Croisille, Les Belles Lettres, Paris.

Jacoby F., 1998, Fragmente der Griechischen Historiker, Brill, Leyde (1921).

Prater A., 2010, «The rediscovery of colour in greek architecture and sculpture », p. 23-36, Circumlitio, Hirmer, München.

Quatremère de Quincy A., 1814, Le Jupiter olympien ou l'Art de la sculpture antique, Firmin Didot, Paris.

Raoul-Rochette D., 1836, Peintures antiques inédites précédées de recherches sur l'emploi de la peinture dans la décoration des édifices sacrés et publics chez les Grecs et chez les Romains, faisant suite aux "Monuments inédits », Imprimerie nationale, Paris.

Strabon, Géographie, 2003, livre XII [t. IX], CUF, trad. F. Lassère, Les Belles Lettres, Paris, [1981]

Stewart J., Revett N., 1762-1816, Antiquities of Athens, J. Haberkorn, Londres.

Tiverios M.A., Tsiafakis D. S., 2002, Color in Ancient Greece, Hydrima Meleton Lampraki, Thessaloniki.

Vitruve, 2003, De l'architecture, VII 9, 2-4, CUF, trad. B. Liou, M. Zuinghedau, Les Belles Lettres, Paris. 